\title{
Extremal graphs having no matching cuts
}

\author{
Paul Bonsma Arthur Marley Andrzej Proskurowski
}

August 5, 2010

\begin{abstract}
A graph $G=(V, E)$ is matching immune if there is no matching cut in $G$. We show that for any matching immune graph $G,|E| \geq\lceil 3(|V|-1) / 2\rceil$. This bound is tight, as we define operations that construct, from a given vertex, exactly the class of matching immune graphs that attain the bound.
\end{abstract}

Keywords: matching cut, matching immune, extremal graph, primitive graph, matching immune

AMS subject classifications: $05 \mathrm{C} 35,05 \mathrm{C} 75$

\section{Introduction}

For basic graph theoretic definitions we refer to [1]. The order of a graph $G=(V, E)$ is $|V|$, and the size of $G$ is $|E|$. For two disjoint sets of vertices $S, T \subset V,[S, T]$ denotes the set of edges with exactly one end vertex in $S$ and one end vertex in $T . M \subseteq E$ is an edge cut in $G$ if $M=[S, \bar{S}]$, where $\bar{S}$ denotes $V \backslash S$, for some $S \subset V, S \neq \emptyset, S \neq V$. If a set $S \subset V$ exists such that $M=[S, \bar{S}]$ and $u \in S$ and $v \in \bar{S}$ then the edge cut $M$ is said to separate $u$ from $v . M \subseteq E$ is a matching if no two edges in $M$ share an end vertex. $M$ is called a matching cut if $M$ is an edge cut and a matching. If a graph has no matching-cut, it is said to be matching immune.

This paper first presents results previously reported in the conference paper [6], namely that for any matching immune graph $(V, E),|E| \geq\lceil 3(|V|-1) / 2\rceil$ holds, and that this bound is tight for every value of $|V|$. This is demonstrated by the class of $A B C$ graphs defined in Section 3, which are matching immune graphs with minimum size for a given order. The paper then presents a proof of the conjecture stated in [6], namely that $\mathrm{ABC}$ graphs are exactly the class of matching immune graphs with minimum size. Some details of this proof appear elsewhere [2, Chapter 4].

A related result appears in [11], where a lower bound is established on the size of connected graphs of given order that cannot be disconnected by removing an independent vertex set. Matching cuts have been studied under other names, including simple cuts, stable cutsets in line graphs, and disconnecting matchings. Matching immune graphs are also called indecomposable graphs. In [4] and [7], primitive graphs are studied, which are matching immune graphs that have no matching immune subgraphs. Graham [7] shows that primitive graphs have applications to the study of cube numberings. Results regarding the complexity of finding matching cuts for different graph classes can be found in [3], [5], [8], [9] and [10]. For many graph classes, deciding whether a member of the class is matching immune is an $\mathcal{N} \mathcal{P}$-hard problem.

\section{A lower bound on the size of matching immune graphs}

In this section, we establish the minimum size for matching immune graphs of a given order. Our proof subjects a given matching immune graph to a series of reduction operations that preserve immunity. Each operation eliminates either three edges and two vertices or at least two edges and one vertex. The operations are expressed in terms of edge contractions and deletions. 


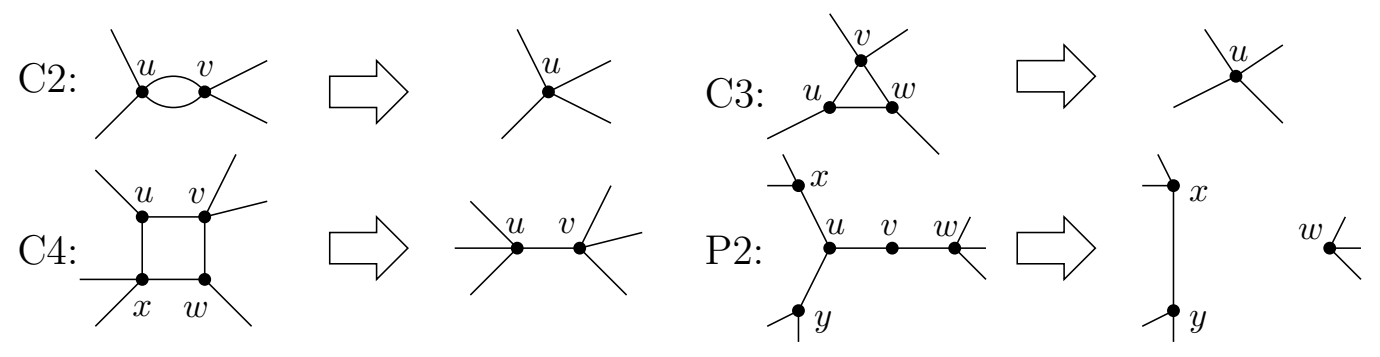

Figure 1: The four reduction operations

For our proofs it is essential to keep track of edge identities, when applying graph operations such as edge contractions. Therefore we mention Bondy and Murty's original definition of a graph, which contains labeled edges [1]: A multi-graph (graph for short) is a triple $G=(V, E, \psi)$, where $V$ and $E$ are disjoint sets representing the vertices and edges, and $\psi$ is an incidence function that associates with each edge of $G$ an unordered pair of (not necessarily distinct) vertices, its end vertices. The deletion of edge $e$ is simply removing $e$ from the set of edges of a graph. The contraction of edge $e$ with end vertices $u_{1}$ and $u_{2}$ is realized by the following three steps: (i) delete edge $e$, (ii) introduce a new vertex $u$, and for every remaining edge having either $u_{1}$ or $u_{2}$ as an end vertex replace that end vertex by $u$ (in the incidence function $\psi$ ), then (iii) delete vertices $u_{1}$ and $u_{2}$ from $G$. Contraction of an edge in a simple graph may result in parallel edges; edge contraction in a graph with parallel edges may result in loops. If a graph $G^{\prime}$ can be obtained from $G$ by a series of edge contractions and edge deletions, then all edges of $G^{\prime}$ are also edges of $G$, although they may have different end vertices in the two graphs. We will often consider one particular edge set $M$ and study its properties both in $G^{\prime}$ and in $G$. For instance, $M$ can be a matching in $G$ but not in $G^{\prime}$. In the remainder of the paper, we will however use the conventional notations again, and omit the incidence function $\psi$ from the definition of a graph. In a slight abuse of notation, edges will be denoted by their end vertices if there is no cause for confusion.

The reduction operations are named after the structure they reduce. Let $C_{n}$ denote a cycle on $n$ vertices $\left(n\right.$-cycle). So by $C_{2}$ we denote a loopless (multi-)graph with two vertices and two edges. $P_{n}$ denotes a path on $n$ vertices. The four operations are illustrated in Figure 1 and are defined as follows:

C2 Suppose there are multiple edges between vertices $u$ and $v$. The $\mathrm{C} 2$ operation consists of deleting all except one of these edges, and contracting the remaining edge.

C3 Let the vertices $u, v$ and $w$ induce a $C_{3}$. The $\mathrm{C} 3$ operation consists of deleting $u v$ and contracting $u w$ and $v w$.

C4 Let subgraph $C$ be a 4 -cycle with edge set $\{u v, v w, w x, u x\}$. The $\mathrm{C} 4$ operation consists of deleting $w x$ and contracting $u x$ and $v w$. Note that for a $C_{4}$ subgraph, the $\mathrm{C} 4$ operation can have two different results.

P2 Let the vertices $u$ and $v$ be neighbors with $d(u)=3$ and $d(v)=2$. Let $v$ have another neighbor $w \neq u$, and let $u$ have another neighbor $x \neq v(x=w$ is allowed $)$. The $\mathrm{P} 2$ operation consists of deleting $u v$ and contracting $u x$ and $v w$.

Lemma 2.1 Suppose $G^{\prime}$ can be obtained from $G$ by a C2, C3, C4 or P2 operation. Then:

- If $G$ is matching immune, then $G^{\prime}$ is matching immune.

- If $|E(G)|=\lceil 3(|V(G)|-1) / 2\rceil$, then $\left|E\left(G^{\prime}\right)\right| \leq\left\lceil 3\left(\left|V\left(G^{\prime}\right)\right|-1\right) / 2\right\rceil$, with equality preserved by the C3, $C_{4}$ and P2 operation.

Proof: We employ the vertex naming conventions established by Figure 1. We prove the first hypothesis by establishing its contrapositive. Since C2 and C3 collapse cycles to a single vertex, if 
$G^{\prime}$ is obtained from $G$ by a $\mathrm{C} 2$ or $\mathrm{C} 3$ operation, then any matching cut $M$ in $G^{\prime}$ is also a matching cut in $G$.

If $G^{\prime}$ results from an application of $\mathrm{C} 4$, then let $u$ be the vertex resulting from the contraction of $u x$, and let $v$ be the vertex resulting from the contraction of $v w$. Any matching cut that does not include $u v$ in $G^{\prime}$ would also be a matching cut in $G$. Consider a matching cut $M$ in $G^{\prime}$ that includes $u v$. In $G$, no edges of $M$ are incident with $x$ or $w$. $M$ is an edge cut in $G-w x$ that separates $x$ from $w$. So adding $x w$ to $M$ yields a matching cut in $G$.

Finally, let us assume that the applied reduction rule was P2 and that $G^{\prime}$ has a matching-cut $M$. If $M$ does not separate $x$ from $y$ then $M$ or $M+u v$ is a matching-cut in $G$. If $M$ separates $x$ from $y$ then w.l.o.g. $x$ and $w$ are not separated by $M$. In this case, removing $x y$ from $M$ and adding $u y$ yields a matching-cut in $G$.

We have shown that the four operations preserve matching immunity. Applying the C3, C4 or P2 operation decreases the number of vertices by two and the number of edges by three, so if one of these operations is applied and $|E(G)|=\lceil 3(|V(G)|-1) / 2\rceil$, then $\left|E\left(G^{\prime}\right)\right|=\left\lceil 3\left(\left|V\left(G^{\prime}\right)\right|-1\right) / 2\right\rceil$. The $\mathrm{C} 2$ operation decreases the number of vertices by one and the number of edges by at least two, so if $|E(G)|=\lceil 3(|V(G)|-1) / 2\rceil$, then $\left|E\left(G^{\prime}\right)\right| \leq\left\lceil 3\left(\left|V\left(G^{\prime}\right)\right|-1\right) / 2\right\rceil$.

A matching immune graph that does not admit application of any of the four reduction operations is called irreducible matching immune. Note that an irreducible matching immune graph has no $C_{2}, C_{3}$, or $C_{4}$ as a subgraph. Furthermore, in such a graph, vertices can be adjacent to a limited number of vertices of degree two.

Lemma 2.2 In an irreducible matching immune graph $G$, any vertex $v$ can be adjacent to at most $d_{G}(v)-2$ vertices of degree two.

Proof: Suppose in an irreducible matching immune graph $G$, a vertex $v$ exists that has at least $d_{G}(v)-1$ neighbors of degree two. Let $N_{2}$ be the set of neighbors of $v$ with degree two. Consider $[S, \bar{S}]$ with $S=\{v\} \cup N_{2}$. Since $G$ has no cycles of length less than five, this is a matching and therefore a matching-cut.

Lemma 2.3 In an irreducible matching immune graph $G$, a vertex of degree two cannot be adjacent to a vertex of degree less than four.

Proof: A matching immune graph $G$ has no vertices of degree one. By Lemma 2.2 no degree two vertices can be adjacent. Finally, a degree two vertex cannot be adjacent to a vertex of degree three since then reduction P2 could have been applied.

The above three lemmas allow us to establish a lower bound on the size-to-order ratio of irreducible matching immune graphs.

Lemma 2.4 An irreducible matching immune graph $G=(V, E)$ either has only one vertex or has a size-to-order ratio of at least 3/2.

Proof: Let $S$ be the set of vertices of degree two and $F$ the set of vertices adjacent to vertices in $S$. For $U \subseteq V(G)$, let $\operatorname{deg}(U)$ be the sum of the degrees of vertices in $U$. Then, by Lemma 2.3, $\operatorname{deg}(F) \geq 4|F|$ and, by Lemma $2.2, \operatorname{deg}(F)-2|F| \geq \operatorname{deg}(S)=2|S|$. These inequalities allow us to establish that $\operatorname{deg}(S)+\operatorname{deg}(F) \geq \operatorname{deg}(S)+2|F|+(|S|+|F|)=3(|S|+|F|)$, and thus

$$
\begin{gathered}
\operatorname{deg}(V)=\operatorname{deg}(S)+\operatorname{deg}(F)+\operatorname{deg}(V \backslash(S \cup F)) \geq \\
3(|S|+|F|)+3(|V|-(|S|+|F|))=3|V| .
\end{gathered}
$$

The size of $G$ is thus at least $3|V| / 2$, as postulated.

Since all of the reduction operations above decrement the size and the order of a graph by a ratio of at least $3 / 2$, we have obtained a lower bound on the minimum size of matching immune graphs of a given order. 
Theorem 2.1 If $G=(V, E)$ is matching immune, then

$$
|E| \geq\lceil 3(|V|-1) / 2\rceil .
$$

Proof: Let $G$ be a matching immune graph with $n$ vertices and $m$ edges, and let successive applications of the reduction rules result in an irreducible matching immune graph $G^{\prime}$ of order $n^{\prime}$ and size $m^{\prime}$. If $n^{\prime}>1$ then

$$
m \geq m^{\prime}+\frac{3\left(n-n^{\prime}\right)}{2} \geq \frac{3 n^{\prime}}{2}+\left(\frac{3 n}{2}-\frac{3 n^{\prime}}{2}\right)=\frac{3 n}{2}>\left\lceil\frac{3(n-1)}{2}\right\rceil
$$

Otherwise, $n^{\prime}=1, m^{\prime}=0$ and $m \geq \frac{3(n-1)}{2}$. Therefore, we have established that $m \geq\left\lceil\frac{3(n-1)}{2}\right\rceil$.

\section{A class of extremal matching immune graphs: ABC graphs}

In this section we show that the lower bound for the size of matching immune graphs stated in the previous section is tight for every value of $|V|$ by defining a class of matching immune graphs that attain this bound. Such graphs are called extremal matching immune. From the results in the previous section, we have the following lemma.

Lemma 3.1 Any extremal matching immune graph can be reduced to $K_{1}$ by a series of C2, C3, C4 or P2 operations.

Proof: Let $G \neq K_{1}$ be extremal matching immune and let $n=|V(G)|$ and $m=|E(G)|$, so $m=\lceil 3(n-1) / 2\rceil$. Since $\lceil 3(n-1) / 2\rceil<3 n / 2$ for every $n, G$ is not irreducible matching immune (Lemma 2.4), thus a C2, C3, C4 or P2 operation can be applied. The resulting graph is again extremal matching immune (Lemma 2.1, Theorem 2.1), so by induction we can continue until $K_{1}$ is obtained.

We now define three operations, A, B, and C, which are limited forms of inverse operations of our reduction operations. These operations will be used to construct a class of extremal matching immune graphs.

Definition 3.1 An A operation on a vertex $u$ introduces two new vertices $v$ and $w$ and the edges $u v, u w$ and $v w$.

$A \mathrm{~B}$ operation on the edge uv introduces two new vertices $w$ and $x$ and the edges uw, vw, $u x$ and $v x$, and removes the edge uv.

$A$ C operation on the vertices $u$ and $v(u=v$ is allowed) introduces a new vertex $w$ and the edges uw and vw.

Note that the $\mathrm{C}$ operation is the only operation that may introduce parallel edges, when $u=v$.

Lemma 3.2 A graph $G^{\prime}$ obtained from a graph $G$ by application of operation $A$ or $B$ is matching immune if $G$ is matching immune. If $|E(G)|=\lceil 3(|V(G)|-1) / 2\rceil$, then $\left|E\left(G^{\prime}\right)\right|=\left\lceil 3\left(\left|V\left(G^{\prime}\right)\right|-\right.\right.$ 1)/27

Proof: Suppose $G^{\prime}$ is obtained by applying operation A to $G$. The new edges are part of a triangle and therefore cannot be part of a matching-cut, so any matching-cut in $G^{\prime}$ is a matching-cut in $G$. Suppose $G^{\prime}$ is obtained by applying a B operation to edge $u v$ of $G$, introducing vertices $w$ and $x$. If $M$ is a matching-cut in $G^{\prime}$ that contains none of the new edges, then $M$ is also a matching-cut in $G$ that does not contain $u v$. If $M$ is a matching-cut in $G^{\prime}$ that contains some of the new edges, then w.l.o.g. $\{u w, v x\} \subseteq M$ and $M$ separates $u$ and $v$. In this case, $M-u w-v x+u v$ is a matching-cut in $G$. These operations add two vertices and three edges, so the relation between the size and order is preserved.

The next lemma can be proved similarly. 
Lemma 3.3 A graph $G^{\prime}$ obtained from a graph $G$ by application of operation $C$ is matching immune if $G$ is matching immune. If $|E(G)|=\lceil 3(|V(G)|-1) / 2\rceil$, then $\left|E\left(G^{\prime}\right)\right|=\left\lceil 3\left(\left|V\left(G^{\prime}\right)\right|-\right.\right.$ 1)/2ך if and only if $|V(G)|$ is odd.

Based on these three operations, we can define $\mathrm{AB}$ graphs and $\mathrm{ABC}$ graphs, named after the operations used in their construction.

Definition 3.2 An AB graph is a graph that can be obtained from $K_{1}$ by a sequence of $A$ and $B$ operations. An $\mathrm{ABC}$ graph is a graph that can be obtained from $K_{1}$ by a sequence of $A$ and $B$ operations and at most one $C$ operation.

From the previous two lemmas, the following corollary follows.

Corollary 3.1 If $G$ is an $A B$ graph on $n$ vertices with $m$ edges, then $n$ is odd, $m=3(n-1) / 2$ and $G$ is matching immune. If $G$ is an $A B C$ graph on $n$ vertices with $m$ edges but $G$ is not an $A B$ graph, then $n$ is even, $m=(3 n-2) / 2=\lceil 3(n-1) / 2\rceil$ and $G$ is matching immune.

So ABC graphs are extremal matching immune graphs. Now, we prove that all extremal matching immune graphs are $\mathrm{ABC}$ graphs.

\section{All extremal matching immune graphs are ABC graphs}

\subsection{An overview of the proof}

In this section, we show that all extremal matching immune graphs are ABC graphs. Our proof is by contradiction, so first we assume an extremal matching immune graph exists that is not an ABC graph. Then we consider a graph $G$ with minimum size among all such graphs. This is called a minimum counterexample.

Theorem 4.1 Every extremal matching immune graph is an ABC graph.

Proof: Suppose this is not true, so there exist counterexamples, which are extremal matching immune graphs that are not ABC graphs. Let $G$ be a minimum counterexample. $G \neq K_{1}$, so by Lemma 3.1, a C2, C3, C4 or P2 operation can be applied to $G$. Below, we exclude these cases one by one: Lemma 4.10 shows that $G$ is simple, so no $\mathrm{C} 2$ operation can be applied. Lemma 4.13 shows that $G$ does not contain a $C_{4}$. Using this information, Lemma 4.12 excludes the case that $G$ contains a $C_{3}$. Finally, Lemma 4.11 then excludes the case that a P2 operation can be applied to $G$. Together, this gives a contradiction, so no counterexample exists.

The four remaining lemmas will be proved in Section 4.4. Some details and cases are omitted, these can be found in [2]. The main idea behind the proofs is as follows: apply the appropriate operation to $G$. This yields a new extremal matching immune graph $G^{\prime}$, with $\left|E\left(G^{\prime}\right)\right| \leq\left\lceil 3\left(\left|V\left(G^{\prime}\right)\right|-1\right) / 2\right\rceil$ (Lemma 2.1), so by Theorem 2.1, $\left|E\left(G^{\prime}\right)\right|=\left\lceil 3\left(\left|V\left(G^{\prime}\right)\right|-1\right) / 2\right\rceil$, and the graph is again extremal matching immune. Since $G$ is a minimum counterexample, $G^{\prime}$ then is an ABC graph. We show that for every possible way of reversing the reduction operations, a graph is obtained that either has a matching-cut, or is $\mathrm{ABC}$, which contradicts that $G$ is a counterexample.

Before we can prove these lemmas, we need to study the structure of ABC graphs in more detail, which is done in Section 4.2. In addition we show that ABC graphs contain many edge cuts that are 'almost' matching-cuts, in Section 4.3.

\subsection{The structure of ABC graphs}

If $G$ is an $\mathrm{AB}(\mathrm{C})$ graph, a sequence of operations that constructs $G$ is called a derivation of $G$. In such a sequence, for every operation it is specified whether it is an $\mathrm{A}, \mathrm{B}$ or $\mathrm{C}$ operation, on which vertex / edge / vertices it works (for the A, B, or C operation respectively), and how the 
newly introduced vertices are labeled. If $k$ operations are applied, this yields a sequence of graphs $G_{0}, \ldots, G_{k}$, with $G_{0}=K_{1}, G_{k}=G$, and where $G_{i}$ is obtained from $G_{i-1}$ by the $i$-th operation in the sequence $(1 \leq i \leq k)$. The graphs $G_{0}, \ldots, G_{k-1}$ are called intermediate graphs in this particular derivation of $G$. Note that an ABC graph generally has many different derivations. We say that a vertex $v$ is used in operation $x$ if $x$ is an $\mathrm{A}$ or $\mathrm{C}$ operation on $v$, or a $\mathrm{B}$ operation on an edge incident with $v$.

Definition 4.1 A graph $G$ that can be obtained from a (labeled copy of) graph $H$ by applying $B$ operations is called an $H$-component.

For an $H$-component $G$, the sequence of B operations that constructs $G$ from $H$ is called a derivation of $G$ from $H$ (or starting with $H$ ). Note that $G$ does not have to be an ABC graph, but it is an ABC graph if $H$ is an ABC graph. $K_{2}, C_{2}, C_{3}$, and $P_{3}$ will often be used for $H$ in the context of $H$-components. A $K_{2}$-component is also called an edge component. If we consider a derivation starting with a $K_{2}$ on vertices $u$ and $v$, then this is called an edge component between $u$ and $v$. $\mathrm{A} C_{3}$-component is also called a triangle component. If we consider a derivation starting with a specific labeled copy of $C_{3}$, then the vertices of this $C_{3}$ are called the triangle vertices of this triangle component. Note that triangle components are AB graphs. Both $C_{2}$ and $P_{3}$-components are associated with the $\mathrm{C}$ operation in a derivation. The end vertices of the $P_{3}$ are called the end vertices of the $P_{3}$-component. Observe that the edges of $\mathrm{ABC}$ graphs can be partitioned into edge induced subgraphs that are all $H$-components for $H=C_{3}, C_{2}$ or $P_{3}$ : A operations introduce $C_{3^{-}}$ components, the $\mathrm{C}$ operation introduces a $C_{2}$ or $P_{3}$-component, and $\mathrm{B}$ operations extend existing $H$-components.

Observation 4.1 For any derivation of an $A B C$ graph $G$, we can partition the edges of $G$ into sets $A_{1}, \ldots, A_{k}$ and at most one set $C$ such that for every $i, G\left[A_{i}\right]$ is a triangle component, and $G[C]$ is a $C_{2}$ or $P_{3}$-component.

Triangle components that correspond to a derivation of $G$ in this way are called $A$-components, and the $C_{2}$ or $P_{3}$-component that corresponds to the $\mathrm{C}$ operation is called the $C$-component. Similar to Observation 4.1, if we have a derivation of $G$ from $H$, then the edges of $G$ can be partitioned into edges components corresponding to the edges of $H$.

Observation 4.2 For any derivation of an $H$-component $G$ from $H$, we can partition $E(G)$ into sets $\left\{E_{u v}: u v \in E(H)\right\}$ such that $G\left[E_{u v}\right]$ is an edge component between $u$ and $v$.

When a particular derivation is chosen, the edge components $G\left[E_{u v}\right]$ from this observation will be denoted as $E C(u v)$. For instance, for a triangle component $G$ with triangle vertices $a, b$ and $c$ (so a particular derivation is chosen), we will often consider the subgraphs $E C(a b), E C(a c)$ and $E C(b c)$. Observe that $E C(a b)$ is an edge component that only shares vertices $a$ and $b$ with other edges in the graph. Such vertices which will be called terminals:

Definition 4.2 Let $H$ be a subgraph of $G$, and $v \in V(H)$. If $d_{H}(v)<d_{G}(v)$ then $v$ is called a terminal of $H . H$ is called an $i$-terminal subgraph of $G$ if $H$ has at most $i$ terminals.

This definition will be used often for 4-cycles and edge components. Note that if $G$ is matching immune and an induced $C_{4}$ is a 2-terminal subgraph of $G$ with terminals $u$ and $v$, then $u$ and $v$ cannot be neighbors, lest the two edges of the $C_{4}$ incident with exactly one of $u$ and $v$ be in a matching-cut. In this case the $C_{4}$ is called a 2-terminal 4-cycle between $u$ and $v$.

We now show that triangle components have many different derivations. In particular, if edge $u v$ is an edge in triangle component $T$, then $T$ has a derivation that starts with a triangle that contains edge $u v$. This is shown in Lemma 4.3.

Lemma 4.1 If $T$ is a triangle component and contains a 2-terminal 4-cycle $C$, then a C4 operation on $C$ yields again a triangle component. 
Proof: Observe that a triangle component $T$ only contains a triangle if it is a triangle. We argue that all edges of the 2-terminal 4-cycle $C$ are introduced by the same B operation, unless $T=K_{2,3}$. Consider the first intermediate graph $G_{i}$ in the derivation that contains all edges of $C$. Let the B operation that yields $G_{i}$ be on edge $u v$, introducing $w$ and $x$. Suppose $C$ is not equal to the newly introduced $C_{4}$. Since it contains some edges of it, it contains exactly two of those, and w.l.o.g. $E(C)=\{u x, v x, u y, v y\}$ for some vertex $y$. But then $G_{i-1}$ contains a triangle on vertices $u, v, y$, and hence is equal to this triangle. In that case $G_{i}$ is a $K_{2,3}$.

Summarizing, $G=C_{3}, G=K_{2,3}$, or all edges of a 2-terminal 4-cycle in $G$ are introduced the same B operation. If $G=C_{3}$, no C4 operation can be applied. If $G=K_{2,3}$, the statement is clear. In the final case, observe that we may obtain an alternative derivation of $G$ by swapping the order of two consecutive B operations, as long as the second B operation does not use vertices that are introduced in the first. Vertices with degree 2 in $G$ are not used in any B operation. Hence we may consider a derivation of $G$ where all edges of $C$ are introduced by the last B operation. Now we see that a $\mathrm{C} 4$ operation on $C$ yields the intermediate graph right before this $\mathrm{B}$ operation is applied, which is also a triangle component.

Lemma 4.2 If $T$ is a triangle component with at least 5 vertices, then for every edge e $\in E(T)$, $T$ contains a 2-terminal 4-cycle that does not contain e.

Proof: Observe that every triangle component on 5 vertices is a $K_{2,3}$, and for this graph the property holds. Now consider a triangle component $T$ with at least 7 vertices, and a derivation of $T$. For an edge $e$ that is not one of the edges introduced by the last B operation, the statement is obvious. Otherwise, consider the triangle component $T^{\prime}$ from which $T$ was constructed by a $\mathrm{B}$ operation on an edge $e^{\prime}$ (so $T^{\prime}$ does not contain $e$ ). By induction, $T^{\prime}$ contains a 2-terminal 4-cycle $C$ that does not contain $e^{\prime}$. After the B operation on $e^{\prime}, C$ still is a 2-terminal 4-cycle in $T$, which proves the statement for $T$. Since triangle components have an odd number of vertices, this proves the claim by induction.

By combining the previous two claims, we obtain a useful Lemma.

Lemma 4.3 If $u$ is a vertex in a triangle component $T$, then a derivation of $T$ exists that starts with the single vertex $u$. If $u v$ is an edge in a triangle component $T$, then a derivation of $T$ exists where $u$ and $v$ are triangle vertices.

Proof: We first prove the statement for the edge $u v$ by induction. For $T=C_{3}$, the statement is clearly true. Otherwise, $T$ contains a 2-terminal 4-cycle $K$ that does not contain edge $u v$ (Lemma 4.2). A C4 operation on $K$ gives another triangle component $T^{\prime}$ (Lemma 4.1), from which $T$ can be constructed (assuming proper vertex labeling in $T^{\prime}$ ). By induction, $T^{\prime}$ has a derivation where $u$ and $v$ are triangle vertices. The statement for the single vertex $u$ follows immediately.

The following lemma describes the block structure of ABC graphs. A block of a graph is a maximal connected subgraph without cut vertices. The edge sets of blocks of $G$ partition $E(G)$, therefore we will also view blocks as edge sets. Considering blocks is useful for our purposes since if $H$ is a block of $G$, then any edge cut of $H$ is also an edge cut of $G$. For basic properties of blocks, see [1].

A operations introduce new blocks, which have odd order. B operations add more edges to existing blocks. That is, for a B operation on $u v$, the four new edges become part of the same block that included $u v$ before. B operations do not change the parity of the block order. For a $\mathrm{C}$ operation on vertices $x$ and $y$ in an $\mathrm{AB}$ graph $G$, that introduces $z$, every path from $x$ to $y$ in $G$ contains edges of the same set of blocks of $G$. The $\mathrm{C}$ operation joins these blocks into one block, together with the new edges $x z$ and $y z$. If all blocks that are joined have odd order, the result is an even order block. These observations show that ABC graphs have the following block structure. (For a detailed proof see [2].) 
Lemma 4.4 An ABC graph $G$ is connected and consists of odd order blocks and at most one even order block. For every derivation of $G$, the odd order blocks are A-components, and the even order block $B$ has the following structure: it contains the $C$-component $C$, and either $B=C$ and $C$ is a $C_{2}$-component, or $C$ is a $P_{3}$-component with (distinct) end vertices $x$ and $y$ such that

1. There are $k \geq 1$ A-components $T_{1}, \ldots, T_{k}$ such that $E(B)=E(C) \cup E\left(T_{1}\right) \cup \ldots \cup E\left(T_{k}\right)$.

2. There are $k-1$ vertices $v_{1}, \ldots, v_{k-1}$ such that $V\left(T_{i}\right) \cap V\left(T_{i+1}\right)=\left\{v_{i}\right\}$, and no other $A$ components in $B$ share vertices.

3. If $k=1$ then $V\left(T_{1}\right) \cap V(C)=\{x, y\}$. If $k \geq 2$ then $V\left(T_{1}\right) \cap V(C)=\{x\}$ and $V\left(T_{k}\right) \cap V(C)=$ $\{y\}$. No no other $A$-components in $B$ share vertices with $C$.

Note that there is an even order block in an ABC graph $G$ if and only if $G$ has even order.

Lemma 4.5 If an $A B C$ graph $G$ is simple, then a derivation of $G$ exists such that every intermediate graph is simple.

Proof: Consider a derivation of a simple ABC graph $G$. The only way that parallel edges can be introduced is with a $\mathrm{C}$ operation on $x=y$, introducing $z$. Since $G$ is simple, one of the edges between $x$ and $z$ must be used in a B operation, that introduces $v$ and $w$. Now instead of the $\mathrm{C}$ operation, use an A operation on $x$ that introduces $v$ and $z$. Instead of the $\mathrm{B}$ operation, use a $\mathrm{C}$ operation on $x$ and $z$ that introduces $w$. This gives the desired derivation of $G$.

\subsection{Edge cuts in $\mathrm{ABC}$ graphs that are almost matching-cuts}

In this section we show various ways to find matching-cuts for edge components, and for graphs deduced from edge components, triangle components and $P_{3}$-components by an expansion operation. A vertex expansion can be seen as the reverse of a contraction. When viewing graphs as tuples $(V, E, \psi)$ where $\psi$ is the incidence function on the edges $E$ (see Section 2), then a vertex expansion of $u$ into $u_{1} u_{2}$ consists of these steps: introducing vertices $u_{1}$ and $u_{2}$, introducing an edge $e$ with $\psi(e)=u_{1} u_{2}$, replacing every occurrence of $u$ in $\psi$ by $u_{1}$ or $u_{2}$, and deleting $u$. Note that there is only one way to contract a particular edge of a graph (apart from the resulting vertex label), but in general there are many ways to expand a vertex into an edge. A vertex expansion of $u$ into $u_{1} u_{2}$ is called a non-trivial vertex expansion if $d\left(u_{1}\right) \geq 2$ and $d\left(u_{2}\right) \geq 2$ in the resulting graph. Suppose $G^{\prime}$ can be obtained from $G$ by a series of edge contractions and edge deletions. Then $G$ can be constructed from $G^{\prime}$ by a series of vertex expansions and edge additions, which motivates the following definition.

Definition 4.3 Suppose $G$ can be obtained from $G^{\prime}$ be a series of vertex expansions and edge additions. Then we say that an edge set $M \subseteq E\left(G^{\prime}\right)$ is split if $G^{\prime}[M]$ and $G[M]$ are not isomorphic. In this case, the subgraph $G^{\prime}[M]$ is also said to be split. Similarly, we say that two edges $e, f \in$ $E\left(G^{\prime}\right)$ are split if $G^{\prime}[\{e, f\}]$ and $G[\{e, f\}]$ are not isomorphic.

Throughout this section, we use $G_{u v}$ to denote an edge component with which we associate a derivation from a copy of $K_{2}$ with vertex labels $u$ and $v$.

Lemma 4.6 For every edge component $G_{u v}$ and every edge $e \in E\left(G_{u v}\right)$, there is a matching-cut $M$ that separates $u$ from $v$ with $e \in M$.

Proof: If $G_{u v}$ consists only of the edge $u v$ then the statement is clearly true. Otherwise, consider a derivation of $G_{u v}$, where the last operation is a B operation on an edge $x y$ in the intermediate graph $G_{u v}^{\prime}$, introducing a 2-terminal 4-cycle $C$ between $x$ and $y . G_{u v}^{\prime}$ is also an edge component, so if $e \in E\left(G_{u v}^{\prime}\right)$, then by induction it has a matching-cut $M$ that contains $e$ and separates $u$ from $v$. $M$ can be turned into a matching-cut in $G_{u v}$ with the desired properties. If $x y \in M$ then this requires replacing $x y$ by two edges of $C$. On the other hand, if $e \notin E\left(G_{u v}^{\prime}\right)$, then $e \in E(C)$. 

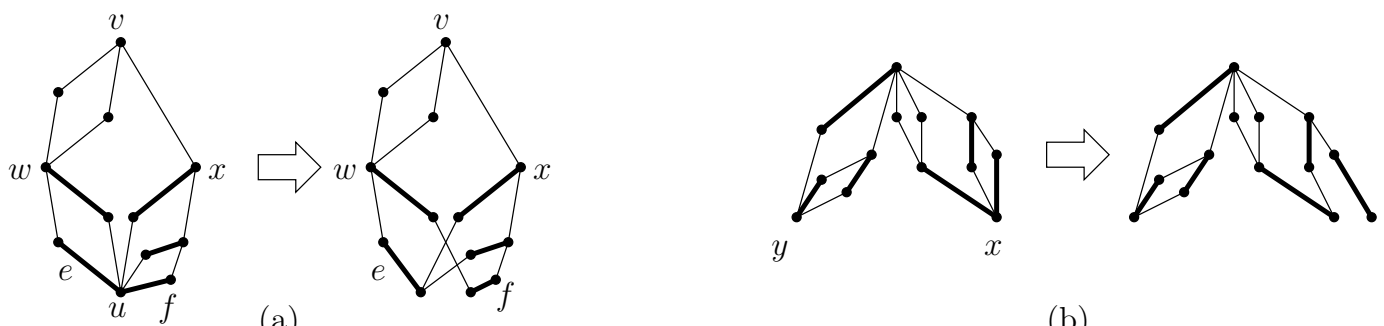

(a)

(b)

Figure 2: Matching-cuts in split $K_{2^{-}}$and $P_{3}$-components.

Consider a matching-cut $M$ for $G_{u v}^{\prime}$ that separates $u$ from $v$ with $x y \in M$, which again exists by induction. $M$ can be turned into a matching-cut $M^{\prime}$ in $G_{u v}$ that separates $u$ from $v$ with $e \in M^{\prime}$.

The proof of the following claim is illustrated in Figure 2(a). In the claim we consider the same edge set $M$ in two different graphs, that can be obtained from each other by contractions resp. vertex expansions.

Lemma 4.7 If a graph $G^{\prime}$ can be constructed from an edge component $G_{u v}$ by a non-trivial vertex expansion of $u$, then there is an edge cut $M$ in $G_{u v}$ that separates $u$ from $v$, is not incident with $v$, and is a matching-cut in $G^{\prime}$.

Proof: Since $G^{\prime}$ is obtained by a non-trivial vertex expansion, in $G_{u v}$ we have $d(u) \geq 2$. So in a derivation of $G_{u v}$ at least one B operation is applied. Let the first B operation introduce two vertices $w$ and $x$. So the edges of $G_{u v}$ can be partitioned into 2-terminal edge components $E C(u w), E C(u x), E C(v w)$ and $E C(v x)$. Since the vertex expansion is non-trivial, we can find $e \in E(E C(u w))$ and $f \in E(E C(u x))$ such that $e$ and $f$ are split by the vertex expansion. Let $M_{1}=\left[S_{1}, T_{1}\right]$ be a matching-cut for $E C(u w)$ with $u \in S_{1}, w \in T_{1}$ and $e \in M_{1}$ (Lemma 4.6). Let $M_{2}=\left[S_{2}, T_{2}\right]$ be a matching-cut for $E C(u x)$ with $u \in S_{2}, x \in T_{2}$ and $f \in M_{2}$. The only adjacent edges in $M_{1} \cup M_{2}$ are $e$ and $f$, so $M_{1} \cup M_{2}$ becomes a matching in $G^{\prime}$. Considering the vertices that the four edge components have in common, we see that $M_{1} \cup M_{2}$ is an edge cut in $G_{u v}$ and therefore also an edge cut in $G^{\prime}$.

The following two lemmas are useful for determining matching-cuts in graphs made from ABC graphs by an expansion operation.

Lemma 4.8 Let $T^{\prime}$ be a graph that can be made from a triangle component $T$ with triangle vertices $u, v$ and $w$ by a non-trivial vertex expansion of $v$. There is an edge cut $M$ in $T$ that is not incident with any vertex in $V(E C(u w)) \backslash\{u, w\}$ and that is a matching-cut in $T^{\prime}$.

Proof: The edges of $T$ can be partitioned into 2-terminal edge components $E C(u v), E C(u w)$ and $E C(v w)$ (Observation 4.2). Since the vertex expansion is non-trivial, we can find $e \in E(E C(u v))$ and $f \in E(E C(v w))$ such that $e$ and $f$ are split by the expansion. Using these two edges, we can find the desired matching-cut similar to the previous proof.

Lemma 4.9 is illustrated in Figure 2(b). Recall that if $\{x, y\} \in S$, we say that the edge cut $M=[S, \bar{S}]$ does not separate $x$ and $y$, even though there may not be an $(x, y)$-path in $G-M$.

Lemma 4.9 Let $P^{\prime}$ be a graph that can be made from a $P_{3}$-component $P$ with end vertices $x$ and $y$ by a non-trivial vertex expansion of $x$. In $P$ an edge cut $M$ exists that does not separate $x$ and $y$ and that is a matching-cut in $P^{\prime}$.

Proof: The edges of $P$ can be partitioned into 1-terminal edge components $E C(x z)$ and $E C(y z)$ that only have $z$ in common. Since $x$ is only incident with edges from $E C(x z)$, the vertex expansion of $P$ corresponds to a non-trivial vertex expansion of $E C(x z)$ into $F^{\prime}$. Now let $M_{1}$ be an edge cut 
for $E C(x z)$ that separates $x$ from $z$, contains no edges incident with $z$, and that is a matching in $F^{\prime}$ (Lemma 4.7). Let $M_{2}$ be any matching-cut for $E C(y z)$ that separates $y$ from $z . M_{1} \cup M_{2}$ forms the desired edge cut. Since $M_{1}$ is a matching in $F^{\prime}$ and contains no edges incident with $z$, this is a matching-cut in $P^{\prime}$.

\subsection{Excluding minimum counterexamples}

Recall that a graph $G$ is a minimum counterexample if it is extremal matching immune, it is not an ABC graph, and has minimum order among all such graphs. Using the properties of ABC graphs deduced in the previous sections, the next four lemmas respectively show that no $\mathrm{C} 2$, P2, C3 or C4 operation can be applied to minimum counterexamples, which together shows that minimum counterexamples do not exist.

Lemma 4.10 A minimum counterexample $G$ is simple.

Proof: If $G$ has vertices $v_{1}$ and $v_{2}$ with at least three parallel edges between them, then one of these edges can be deleted and the resulting graph is still matching immune, contradicting Theorem 2.1. Now suppose there are two parallel edges between $v_{1}$ and $v_{2}$. A C2 operation gives an ABC graph $G^{\prime}$. Let $v \in V\left(G^{\prime}\right)$ be the vertex resulting from the contraction. Since a C2 operation decreases the order and size by one and two respectively, and both graphs are extremal matching immune, it follows that $G^{\prime}$ has odd order and thus is an AB graph. If we reverse the $\mathrm{C} 2$ operation, $G$ can be constructed from $G^{\prime}$ with one vertex expansion and an edge addition. So we can use the terminology of Section 4.3 and consider whether components of $G^{\prime}$ are split or not by the expansion operation.

First assume some A-component $T$ is split. Then a matching-cut for $G$ can be obtained: Since a derivation of $T$ exists such that $v$ is a triangle vertex (Lemma 4.3), Lemma 4.8 shows that an edge cut $M$ exists in $T$ that becomes a matching after the first vertex expansion. Since $T$ is a block in $G^{\prime}$ (Lemma 4.4), $M$ is also an edge cut in $G^{\prime}$, and therefore an edge cut and matching-cut in $G$.

So now we may assume that no A-component is split. We complete the proof by showing how an ABC derivation of $G$ can be obtained from the derivation of $G^{\prime}$. Let $G_{i}$ be the first intermediate graph in the the derivation of $G^{\prime}$ that contains $v$. In $G_{i}$, all edges incident with $v$ are part of the same A-component $T$ of $G^{\prime}$. (Or, in the trivial case, $v$ is the initial vertex and has degree 0 at this point.) Since this triangle component $T$ is not split, in $G$ it is incident with exactly one vertex of $v_{1}$ and $v_{2}$, say $v_{1}$. (More precisely: in $G$, no edge of $E(T)$ is incident with $v_{2}$.) Now change the derivation as follows: instead of introducing $v$ at this point in the derivation, introduce $v_{1}$, and immediately after $v_{1}$ is introduced, apply a $\mathrm{C}$ operation on $v_{1}$ introducing $v_{2}$, and two parallel edges between them. Continue with the derivation as before, except when the derivation calls for an A operation on $v$. In this case, the A-component of $G^{\prime}$ that corresponds to this operation is not split, and therefore in $G$ is incident with exactly one of $v_{1}, v_{2}$. Apply the A operation on this vertex instead. This gives an $\mathrm{ABC}$ derivation of $G$, a contradiction.

Lemma 4.11 If a minimum counterexample $G$ contains no $C_{3}$ or $C_{4}$, then no P2 operation can be applied to $G$.

Proof: Suppose a P2 operation can be applied to $G$. Then in $G$ there are neighbors $u$ and $v$, with $d(u)=3$ and $d(v)=2$. $x$ and $y$ are the other neighbors of $u$, and $z$ is the other neighbor of $v$. If $z=x$ or $z=y$ then $G$ contains a $C_{3}$, a contradiction. If $z$ is adjacent to $x$ or $y$, then $G$ contains a $C_{4}$, also a contradiction. So after the P2 operation is applied, a graph $G^{\prime}$ is obtained that contains edge $x y$ and vertex $z$, and $z$ is not equal to or adjacent to $x$ or $y$. $G^{\prime}$ is an $\mathrm{ABC}$ graph. Clearly, $G^{\prime}$ cannot be a $K_{1}$, a $C_{2}$ or a $C_{3}$. For every ABC graph $G^{\prime}$ other than these three graphs and every choice of edge $x y \in E\left(G^{\prime}\right)$, it is easily checked that there is a $C_{3}$ or $C_{4}$ that does not contain $x y$ (using Lemma 4.2). This corresponds to a $C_{3}$ or $C_{4}$ in $G$, a contradiction. 
Lemma 4.12 If a minimum counterexample $G$ contains no $C_{4}$, then $G$ contains no $C_{3}$.

Proof: Suppose $G$ is a minimum counterexample that does not contain a $C_{4}$, and let $K$ be a triangle in $G$ on vertices $v_{1}, v_{2}$ and $v_{3}$. Applying operation $\mathrm{C} 3$ on $K$ such that the resulting vertex is vertex $v$ gives an ABC graph $G^{\prime}$. Consider a derivation of $G^{\prime}$ with A-components $T_{1}, \ldots, T_{k}$, and if the order of $G^{\prime}$ is even, C-component $P$. The edge sets of these components induce the components $T_{1}^{\prime}, \ldots, T_{k}^{\prime}$ resp. $P^{\prime}$ in $G$. If the order of $G^{\prime}$ is even, one C operation is used in every derivation of $G^{\prime}$. In the derivation we consider, $x$ and $y$ will denote the vertices in $G^{\prime}$ on which the $\mathrm{C}$ operation is applied, and $z$ will denote the vertex introduced by the $\mathrm{C}$ operation. So the C-component $P$ consists of edge components $E C(x z)$ and $E C(y z)$. We choose a derivation of $G^{\prime}$ such that if $G^{\prime}$ is simple, then $x \neq y$, which is possible by Lemma 4.5.

CASE 1: no A-component or C-component of $G^{\prime}$ is split.

The proof is analog to the same case in the proof of Lemma 4.10: we change the derivation of $G^{\prime}$ by, instead of introducing vertex $v$ at some point, introducing the appropriate vertex of $K$, say $v_{1}$, and immediately afterwards introducing $v_{2}$ and $v_{3}$ using an A operation on $v_{1}$. Because no A-components or $\mathrm{C}$-components are split, later $\mathrm{A}$ operations or $\mathrm{C}$ operations on $v$ can be replaced by the same operation on the appropriate $v_{i}$ instead. This gives an $\mathrm{ABC}$ derivation of $G$, a contradiction.

CASE 2: An A-component $T_{i}$ is split, which does not contain both $x$ and $y$ with $x \neq y$.

Let $T_{i}$ be an A-component that is split, so $v \in V\left(T_{i}\right)$. Analog to the proof of Lemma 4.10, an edge cut $M$ in $T_{i}$ can be constructed that is a matching-cut in $T_{i}^{\prime}$ (Lemma 4.8). Observe that $M$ is a matching-cut in $G$ if and only if it is an edge cut in $G^{\prime}$. If $G^{\prime}$ is odd, then $T_{i}$ is a block of $G^{\prime}$ (Lemma 4.4), so $M$ is also an edge cut in $G^{\prime}$. So $G^{\prime}$ is even, and $T_{i}$ is not a block of $G^{\prime}$ and therefore $x \neq y$ in $G^{\prime}$. Suppose that in $G^{\prime}, x$ is not incident with edges from $M$. Consider any matching-cut $M^{\prime}$ in $E C(x z)$ that separates $x$ from $z$. Since edges from $M$ and $M^{\prime}$ share no end vertices, either $M$ or $M \cup M^{\prime}$ is an edge cut in $G^{\prime}$ (Lemma 4.4), and therefore a matching-cut in $G$. A similar matching-cut can be constructed if $y$ is not incident with edges from $M$. We have now found a matching-cut in all cases, except when $M$ separates $x$ and $y$ and is incident with both $x$ and $y$, which concludes case 2 .

CASE 3: The C-component $P$ is split.

Case 3.1: $x=y$. Note that no A-component is split: since $x=y$, this case would be covered by Case 2. Recall that we chose the derivation such that $x=y$ only holds if $G$ is not simple, so since $x=y$ the C-component $P$ consists only of two parallel edges between $x=y$ and a vertex $z$. Since $P$ is split, this means that $v=x$ or $v=z$.

If $v=x$ then $v$ is introduced before the $\mathrm{C}$ operation is applied. At this point $v$ is part of a unique A-component $T$, which is incident with only one of the vertices $v_{1}, v_{2}, v_{3}$ in $G$, say $v_{1}$, since $T$ is not split. Now instead of introducing $v$, introduce $v_{1}$, and immediately apply an A operation introducing $v_{2}$ and $v_{3}$. Continue with the derivation, where A operations on $v$ are replaced with A operations on the appropriate vertex $v_{k}$. The $\mathrm{C}$ operation is now applied on the appropriate two vertices $v_{i}$ and $v_{j}$ (introducing a $P_{3}$ ) instead of only on $v$ (introducing a $C_{2}$ ). Because no Acomponent is split, this $\mathrm{ABC}$ derivation constructs $G$, a contradiction. If $v=z$ then we similarly obtain a derivation of $G$ by replacing the $C$ operation by an A operation introducing $v_{i}$ and $v_{j}$, and immediately afterwards applying a $\mathrm{C}$ operation on $v_{i}$ and $v_{j}$.

Case 3.2: $v \neq x, v \neq y$ and $x \neq y$. Consider the operation that introduces $v$ in the derivation of $G^{\prime}$. If $v=z$ this is the $\mathrm{C}$ operation, otherwise a $\mathrm{B}$ operation on an edge of the C-component. In both cases, after this operation, $v$ is incident with exactly two edges $a v$ and $b v$, with $a \neq b$ (since $x \neq y)$. We consider the edge components $E C(a v)$ and $E C(b v)$ of $G^{\prime}$, and the even order block $B$ of $G^{\prime}$ that contains these. $E C(a v)$ and $E C(b v)$ together form a $P_{3}$-component $Q$, which only has terminals $a$ and $b$ when viewed as a subgraph of $B$. Since $P$ is split we can find edges $e \in E C(a v)$ and $f \in E C(b v)$ both incident with $v$, that are not adjacent in $G$. Let $M_{1}$ be a matching-cut in $E C(a v)$ that contains $e$ and separates $a$ and $v$, and let $M_{2}$ be a matching-cut in $E C(b v)$ that contains $f$ and separates $b$ and $v$ (Lemma 4.6). Together these form an edge cut $M_{1} \cup M_{2}$ for $Q$ that does not separate $a$ and $b$. Since $a$ and $b$ are the only terminals of $Q$ in $B$, this is also an edge 
(a) $T$ :

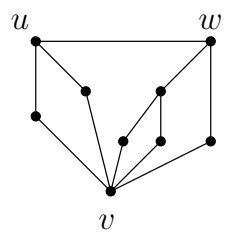

(b) $T^{\prime}$

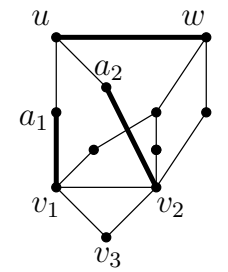

(c) $T^{\prime}$ :

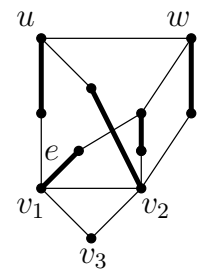

(d) $T^{\prime}$ :

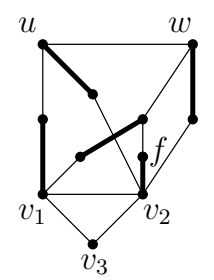

Figure 3: Triangle component $T$, and the three matching-cuts from Case 4.

cut in the block $B$, and therefore in $G^{\prime}$. So by choice of the edges $e$ and $f$, this is a matching-cut in $G$.

Case 3.3: $v=x$ and $x \neq y$. Since $x \neq y$ and $P$ is split, $E C(x z)$ is split. Now Lemma 4.9 shows there is an edge cut in $P$ that does not separate $x$ from $y$, that is a matching-cut in $P^{\prime}$. By the block structure of $\mathrm{ABC}$ graphs (Lemma 4.4), this is a matching-cut in $G$, a contradiction.

Observe that Case 3.1, 3.2 and 3.3 w.l.o.g. cover all possibilities when the C-component is split, so this concludes Case 3.

Summarizing these three cases, we know that some component is split, by the argument in Case 1. Case 3 shows that this is not the C-component, so an A-component $T_{i}$ is split, which then contains both $x$ and $y$, and $x \neq y$ by Case 2 . This is handled in the final case. Here we will use the assumption that $G$ contains no $C_{4}$ for the first time.

CASE 4: An A-component $T_{i}$ is split, which contains $x$ and $y$ with $x \neq y$, and the Ccomponent is not split.

Let $T=T_{i}$. If the $\mathrm{C}$-component contains a $C_{4}$, then since it is not split, $G$ contains a $C_{4}$ as well. But we assumed this is not the case, so the C-component consists only of the edges $x z$ and $y z$. Similarly, every $C_{4}$ in $G^{\prime}$ is split and therefore incident with $v$. In addition, it follows that $T$ is not a triangle, since then the three edges of $T$ together with one edge from $E(K)=\left\{v_{1} v_{2}, v_{1} v_{3}, v_{2} v_{3}\right\}$ would form a $C_{4}$ in $G$. Consider $T^{\prime}=G[E(K) \cup E(T)]$. We will describe three matching-cuts for $T^{\prime}$, and show that no matter how $x$ and $y$ are chosen, one of these matching-cuts is also a matching-cut in $G$. The proof is illustrated in Figure 3.

Since $T$ is not a triangle, in a derivation of $T$, at least one $\mathrm{B}$ operation is used. Consider the last B operation. The 2-terminal 4-cycle corresponding to this operation is split so it has terminals $v$ and another vertex $u$. Let $a_{1}$ and $a_{2}$ be the other vertices of this 2-terminal 4-cycle. There is a derivation of $T$ with triangle vertices $u$ and $v$ (Lemma 4.3). Let $w$ be the third triangle vertex.

W.l.o.g. we assume that $a_{1}$ is adjacent to $v_{1}$ in $T^{\prime}$, and $a_{2}$ is adjacent to $v_{2}$ in $T^{\prime}$. Now we can define the first matching-cut in $T^{\prime}: E C(u w)$ must be a single edge (otherwise $T^{\prime}$ contains a $C_{4}$ ). So in $T^{\prime}$, edge set $M_{1}=\left\{u w, a_{1} v_{1}, a_{2} v_{2}\right\}$ is a matching-cut (see Figure 3(b)).

Next, we argue that $E C(v w)$ contains an edge $e$ that is incident with $v$ in $T$, but not with $v_{2}$ in $T^{\prime}$. If $E C(v w)$ is a single edge, this is true since otherwise $T^{\prime}$ contains a $C_{4}$ (recall that $E C(u w)$ is also a single edge). Otherwise, $E C(v w)$ must be split, and the statement is clear. Similarly, $E C(v w)$ contains an edge $f$ that is incident with $v$ in $T$, but not with $v_{1}$ in $T^{\prime}$.

Using $e$ and $f$ we can find matching-cuts $M_{1}^{\prime}$ and $M_{2}^{\prime}$ of $E C(v w)$ (Lemma 4.6) such that $M_{1}=M_{1}^{\prime} \cup\left\{u a_{1}, v_{2} a_{2}\right\}$ and $M_{2}=M_{2}^{\prime} \cup\left\{u a_{2}, v_{1} a_{1}\right\}$ are matching-cuts in $T^{\prime}$ (see Figure 3(c) and (d)).

If $M$ is a matching-cut in $T^{\prime}$, then at least one of $M, M \cup\{x z\}$ or $M \cup\{y z\}$ is a matching-cut in $G$ unless $M$ separates $x$ from $y$ and $x$ and $y$ are both incident with edges from $M$. Since $T$ contains no $C_{4}$, it is not possible that $x=u$ and $y=v$ (or vice versa). In all other cases, it can be seen that at least one of $M_{1}, M_{2}$ or $M_{3}$ yields a matching-cut in $G$ this way, which concludes the proof of this case.

Now we have considered all cases, so we have shown that it is not possible that a minimum counterexample contains a $C_{3}$ but not a $C_{4}$, which proves the lemma. 
Lemma 4.13 A minimum counterexample $G$ contains no $C_{4}$.

Proof: The proof is very similar to that of Lemma 4.12. The C4 operation yields an ABC graph $G^{\prime}$. We consider all possible results of applying a reversed C4 operation to an ABC graph $G^{\prime}$. If this reversed $\mathrm{C} 4$ operation on edge $u v$ corresponds to a B operation in the A-component or C-component that contains $u v$, and no other A-component or C-component is split, an ABC derivation of $G$ is easily obtained. Otherwise, we can identify a matching-cut in $G$ by considering matching-cuts in split components, or give a derivation of $G$. For details see [2].

\section{Discussion}

We showed that for all matching immune graphs $G=(V, E),|E| \geq\lceil 3(|V|-1) / 2\rceil$ holds. In addition, we proved that all matching immune graphs with $|E|=\lceil 3(|V|-1) / 2\rceil$ are ABC graphs. To prove this, we first deduced a number of properties of $\mathrm{ABC}$ graphs, related to derivations, block structure and matching-cuts in subgraphs of ABC graphs (Sections 4.2 and 4.3).

ABC graphs can be recognized in polynomial time (see [2] for details). Since we showed that these are exactly the matching immune graphs with $|E|=\lceil 3(|V|-1) / 2\rceil$, the class of graphs with this relation between order and size is another class for which immunity can be determined efficiently, in addition to e.g. graphs with maximum degree 3 [5], claw-free graphs and graphs of bounded treewidth [3].

Graham [7] has investigated a class of matching immune graphs which have no matching immune subgraphs, called primitive graphs. He gave a construction procedure defining an infinite class of primitive graphs. In this procedure, two primitive graphs are combined into a new, larger primitive graph. This procedure is related to ABC graphs in the following way. All triangle components can be constructed using this procedure, and therefore are primitive graphs. If only the triangle is taken as a basic primitive graph, all primitive graphs that can be constructed with the procedure are triangle components. In [7], a number of interesting questions on primitive graphs are stated. Some were answered in [4], and the others are still open to our knowledge.

\section{Acknowledgements}

The authors would like to thank Hajo Broersma for helpful discussions and detailed comments on earlier versions of this paper.

\section{References}

[1] J. A. Bondy and U. S. R. Murty. Graph theory, volume 244 of Graduate Texts in Mathematics. Springer, New York, 2008.

[2] P. Bonsma. Sparse cuts, matching-cuts and leafy trees in graphs. PhD thesis, University of Twente, Enschede, the Netherlands, 2006. http://purl.org/utwente/57117.

[3] P. Bonsma. The complexity of the matching-cut problem for planar graphs and other graph classes. Journal of Graph Theory, 62(2):109-126, 2009.

[4] I. Z. Bouwer and G. F. LeBlanc. A note on primitive graphs. Canadian Mathematical Bulletin, 15:437-440, 1972.

[5] V. Chvátal. Recognizing decomposable graphs. Journal of Graph Theory, 8:51-53, 1984.

[6] A. M. Farley and A. Proskurowski. Extremal graphs with no disconnecting matching. In Proceedings of the second West Coast conference on combinatorics, graph theory, and computing (Eugene, 1983), volume 41 of Congressus Numerantium, pages 153-165, 1984. 
[7] R. L. Graham. On primitive graphs and optimal vertex assignments. Annals of the New York academy of sciences, 175:170-186, 1970.

[8] V. B. Le and B. Randerath. On stable cutsets in line graphs. Theoretical Computer Science, 301:463-475, 2003.

[9] A. M. Moshi. Matching cutsets in graphs. Journal of Graph Theory, 13(5):527-536, 1989.

[10] M. Patrignani and M. Pizzonia. The complexity of the matching-cut problem. In Graphtheoretic concepts in computer science (Boltenhagen, 2001), volume 2204 of Lecture Notes in Computer Science, pages 284-295, 2001.

[11] D. J. Rose. On simple characterization of $k$-trees. Discrete mathematics, 7:317-322, 1974. 